\title{
Multiwall carbon nanotube polyvinyl alcohol-based saturable absorber in passively Q-switched fiber laser
}

\author{
H. Ahmad, ${ }^{1, *}$ M. F. Ismail, ${ }^{1}$ S. N. M. Hassan, ${ }^{1}$ F. Ahmad, ${ }^{2}$ \\ M. Z. Zulkifli, ${ }^{1}$ and S. W. Harun ${ }^{1}$ \\ ${ }^{1}$ Photonics Research Center, Department of Physics, University of Malaya, 50603 Kuala Lumpur, Malaysia \\ ${ }^{2}$ Department of Electrical Engineering UTMspace, Universiti Teknologi Malaysia, 54100 Kuala Lumpur, Malaysia \\ *Corresponding author: harith@um.edu.my
}

Received 21 July 2014; revised 10 September 2014; accepted 10 September 2014; posted 11 September 2014 (Doc. ID 217211); published 15 October 2014

\begin{abstract}
In this work, we demonstrated a compact $Q$-switched erbium-doped fiber laser capable of generating high-energy pulses using a newly developed multiwall carbon nanotube (CNT) polyvinyl alcohol (PVA) thin film based saturable absorber. $Q$-switched pulse operation is obtained by sandwiching the thin film between two fiber ferrules forming a saturable absorber. A saturable absorber with 1.25 wt. $\%$ of PVA concentration shows a consistency in generating pulsed laser with a good range of tunable repetition rate, shortest pulse width, and produces a high pulse energy and peak power. The pulse train generated has a maximum repetition rate of $29.9 \mathrm{kHz}$ with a corresponding pulse width of $3.49 \mu \mathrm{s}$ as a function of maximum pump power of $32.15 \mathrm{~mW}$. The maximum average output power of the $Q$-switched fiber laser system is $1.49 \mathrm{~mW}$, which translates to a pulse energy of $49.8 \mathrm{~nJ}$. The proposed method of multiwall CNT/PVA thin film fabrication is low in cost and involves uncomplicated processes.

(C) 2014 Optical Society of America

OCIS codes: (060.2410) Fibers, erbium; (140.3540) Lasers, Q-switched; (140.3560) Lasers, ring; (140.3538) Lasers, pulsed; (060.3510) Lasers, fiber.

http://dx.doi.org/10.1364/AO.53.007025
\end{abstract}

\section{Introduction}

Of late, the growth in carbon nanotubes (CNTs) implementation for fiber laser systems in producing a stable pulse laser has attracted significant research interest. The optical characteristics of the CNT in nonlinear saturable absorption with ultrafast recovery time and wide bandwidth operation [1-4] indicate its potential optoelectronics applications, such as communications, photovoltaic devices, transceiver devices, microelectronics, and passive optical devices. For instance, in passive optical device applications, employment of a CNT-based saturable absorber in

$1559-128 \mathrm{X} / 14 / 307025-05 \$ 15.00 / 0$

(C) 2014 Optical Society of America the laser cavity with appropriate configuration facilitates a stable and least peak-to-peak fluctuation of the pulse train in repetitive $Q$-switched operation.

A repetitive $Q$-switched pulse can be realized either actively or passively. In active $Q$-switching, active loss modulation is achieved by incorporating an acoustic or electro-optic modulator into the laser cavity to trigger pulse train formation. Compared to the active ones, passive $Q$-switching replaces the active modulator with a passive device such as a saturable absorber as a switching device $[5,6]$ in order to obtain higher pulse energy. Moreover, the complexity of the fiber laser system can be drastically reduced.

Single-wall carbon nanotube (SWCNT)-based saturable absorbers have gained great research interest due to their compactness, low saturation 
intensity, and broad bandwidth [7-11]. However, recently an alternative CNT-based saturable absorber, which is a multiwall carbon nanotube (MWCNT)based saturable absorber has also been studied due to its intrinsic properties [12]. In comparison to SWCNTs, the higher mass density of MWCNTs and their multilayer outer walls tube structure influence the absorption of photon per nanotubes, which enhance their ability to generate intense pulses $[13,14]$. This ability is further assisted by having a higher thermal damage threshold than the SWCNTs, as reported in [13], whereby a damage threshold of 15.5 and $7.2 \overline{\mathrm{KW}} / \mathrm{cm}^{2}$ is achieved for MWCNTs and SWCNTs, respectively. Ahmad et al. [15] demonstrated an easy fabrication of MWCNTspolyethylene oxide (PEO) polymer composites as a passive saturable absorber with reduced cost compared to the use of SWCNTs polymer composites. The recovery time of the MWCNTs is about 200 $300 \mathrm{fs}[16,17]$, which is longer than the reported recovery time of SWCNTs. In a repetitively $Q$ switching regime, usually the pulse width is longer than the saturable absorber recovery time, thus it is still worth investigating the performance of MWCNT-based polymer composites as passive saturable absorbers in the erbium-doped fiber laser (EDFL). The MWCNT-based saturable absorber has been demonstrated in the mode-locking regime in a solid-state laser $[16,17]$ and recently in the $Q$-switching regime in a fiber laser [15]. The performance of MWCNTs-PEO-based thin film in a $Q$-switched fiber laser as reported by [15] with the maximum pulse energy of $15.3 \mathrm{~nJ}$ and shortest pulse width produced is around $4.5 \mu \mathrm{s}$.

In this work, we propose and demonstrate the fabrication of a MWCNT polymer composites thin film based saturable absorber with new host material, polyvinyl alcohol (PVA) in a passively $Q$ switched fiber laser system. In comparison to PEO, PVA has its own intrinsic feature, such as larger molecular weight distribution, which increases the mechanical strength of the fabricated thin film. Apart from that, PVA has higher diffusivity resulting in better uniformity in thin film formation [18] and smoother surface MWCNTs polymer composites thin film. The newly developed MWCNT-PVA-based saturable absorber is capable of producing higher pulse energy of $49.8 \mathrm{~nJ}$ at the repetition rate of $29.9 \mathrm{kHz}$ with corresponding pulse width as narrow as $3.49 \mu \mathrm{s}$.

\section{Fabrication of PVA Host Polymer}

The diameter and length of the used MWCNT are approximately $10-20 \mathrm{~nm}$ and $1-2 \mu \mathrm{m}$, respectively. At first, the PVA is functionalized so that it becomes water soluble. It is prepared by dissolving $4 \mathrm{~g}$ of sodium dodecyl sulfate in $400 \mathrm{ml}$ deionized water. A total of $250 \mathrm{mg}$ of MWCNT powder is added into the solution and the mixed solution is sonicated for $60 \mathrm{~min}$ at $50 \mathrm{~mW}$ in order to achieve homogeneous dispersion of the MWCNT. The solution is then centrifuged at $1000 \mathrm{RPM}$ (revolution per minute) to remove large particles of undispersed MWCNT to attain dispersed suspension, which is still stable for weeks of storage.

The PVA is prepared by dissolving $1 \mathrm{~g}$ of PVA $\left(\mathrm{Mw}=89 \times 10^{3} \mathrm{~g} / \mathrm{mol}\right)$ in $120 \mathrm{ml}$ of deionized water with the aid of magnetic stirrer. The MWCNT-PVAbased saturable absorber is fabricated by mixing the dispersed MWCNTs suspension to the PVA solution in a concentration of 1.25 wt. \%. The mixture of MWCNTs and PVA then undergoes an ultrasonication process for $1 \mathrm{~h}$ to obtain a homogenous MWCNT-PVA polymer composite suspension, which is then poured on a Petri dish and then dried in the ambient temperature for one week to develop a MWCNT-PVA-based thin film with a thickness of $50 \mu \mathrm{m}$. The fabricated CNT-based saturable absorber thin film is cut into a small piece by $2 \mathrm{~mm} \times 2 \mathrm{~mm}$ and attached to the fiber ferrule with the aid of an index matching gel before being sandwiched between the other clean fiber ferrule using a connector. Measured insertion loss of the fabricated saturable absorber is around $5 \mathrm{~dB}$ at $1550 \mathrm{~nm}$.

The dispersion state of MWCNTs in the PVA matrix is detected by using field emission scanning electron microscopy (FESEM) with brand name of FE-SEM and model number of JEOL 7600F. Figure 1 shows the FESEM image of the newly developed cross section of the MWCNT-PVA film. As observed, the MWCNTs are clearly well-dispersed in the PVA matrix. In this work, the concentration of MWCNT used is 1.25 wt. \%. The FESEM image shown in Fig. 1 does not reveal the structure of MWCNT distinctly due to the high concentration of PVA polymer. As can be seen from the figure, the circle-like structure could probably be the round end nanotube observed in a cross-sectional view. Figure 2 shows the Raman spectrum of pure PVA film and MWCNTs-PVA-based film. The Raman spectrum of pure PVA film is intense at $2915 \mathrm{~cm}^{-1}$. As observed, the Raman spectrum of MWCNTs-PVA-based film consists of various peaks at $1351 \mathrm{~cm}^{-1}, 1591 \mathrm{~cm}^{1}$, and $2710 \mathrm{~cm}^{-1}$, which correspond to D, G, and G' bands. The Raman spectroscopy of MWCNTs-PVA film is taken at $532 \mathrm{~nm}$ $(2.33 \mathrm{eV})$ excitation in conjunction with 1800 lines/ $\mathrm{mm}$ grating. The $\mathrm{D}$ band at $1351 \mathrm{~cm}^{-1}$ originates from a double resonance process and is attributed

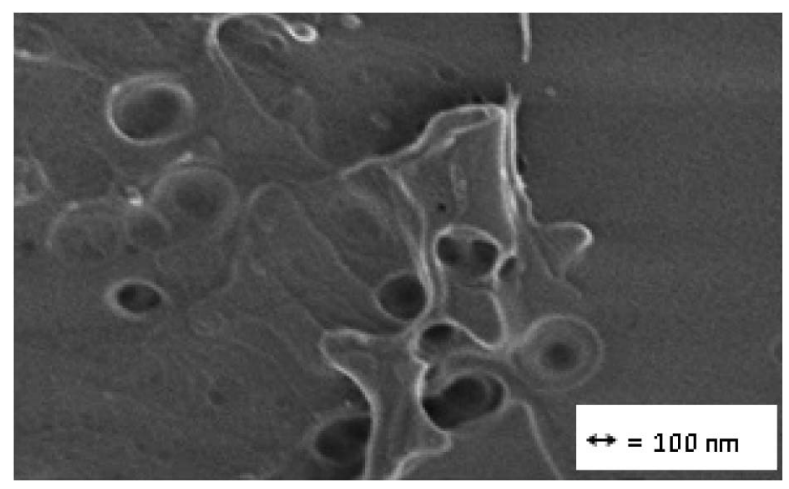

Fig. 1. FESEM image of a MWCNTs-PVA film. 


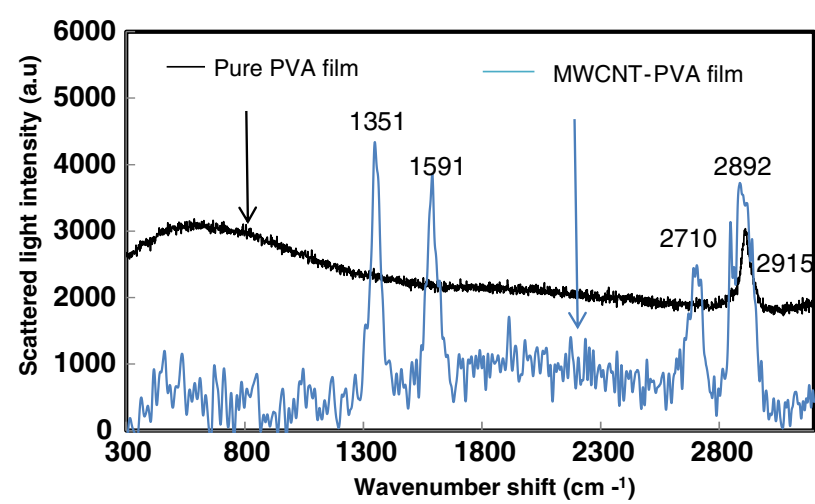

Fig. 2. Unpolarized room temperature Raman scattering spectra of a pure PVA film (black curve) and MWCNTs-PVA film (blue curve).

to the presence of amorphous disordered carbon. The $\mathrm{G}$ band at $1591 \mathrm{~cm}^{-1}$ corresponds to tangential stretching carbon-carbon (C-C) vibrations on the nanotube wall plane $[19,20]$. The $G$ ' peak at $2710 \mathrm{~cm}^{-1}$ originates from two-phonon scattering phenomena around the $\mathrm{K}$ point and around the $\mathrm{M}$ point in the Brillouin zone [21]. The intense peak at $2892 \mathrm{~cm}^{-1}$ is due to the PVA host polymer. Apart from that, the radial breathing mode, which occurs at $100-500 \mathrm{~cm}^{1}$ for SWCNTs, is not observed due to restriction caused by the outer tubes of the MWCNT.

\section{Experimental Setup}

Figure 3 shows the $Q$-switched fiber laser configuration, which employs the newly developed MWCNTPVA-based saturable absorber. It consist of $4 \mathrm{~m} \mathrm{EDF}$ as the gain medium, which is pumped by a $1480 \mathrm{~nm}$ laser diode through a $1480 \mathrm{~nm}$ port of a fused 1480/ 1550 wavelength division multiplexer (WDM). Then the EDF is connected to the input port of a polarization-dependent isolator, which is an optical isolator to ensure the unidirectional oscillation within the cavity. A polarization controller (PC) is incorporated in the laser resonance cavity to optimize the $Q$ switching operation. The propagating light encounters the saturable absorber formed by sandwiching

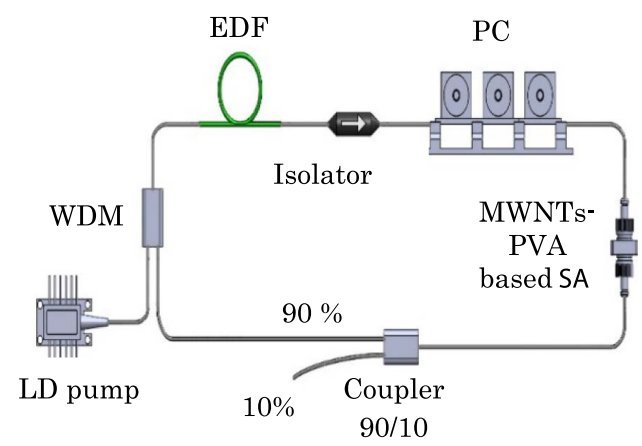

Fig. 3. Schematic configuration of the $Q$-switched EDFL using concentration of 1.25 wt. \% of MWCNTs-PVA. LD, laser diode; WDM, wavelength division multiplexer; EDF, erbium-doped fiber; PC, polarization controller; MWCNT-PVA, multiwall carbon nanotube-poly(vinyl) alcohol; SA, saturable absorber.

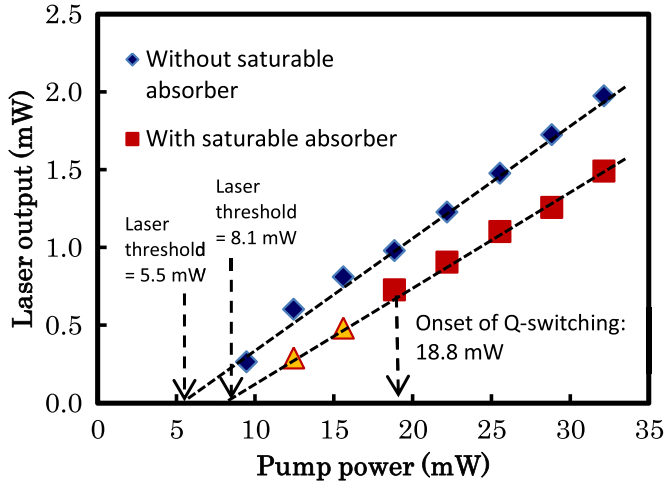

Fig. 4. Lasing output power against the laser diode pump power at $1480 \mathrm{~nm}$, giving slope efficiency of $6.2 \%$ and $7.4 \%$ for without and with saturable absorber.

the MWCNTA-PVA polymer composites in between two PC/FC connectors, where $Q$-switched pulses are generated.

After passing through the MWCNT-PVA saturable absorber, $10 \%$ of the laser output is extracted by the of the $90 / 10$ optical coupler, whereby $90 \%$ of the oscillating laser is fed back into the laser via the WDM $1480 \mathrm{~nm}$ port. Spectral and temporal profiles of the output spectrum are measured by a Yokogawa AQ6317 optical spectrum analyzer with the resolution of $0.02 \mathrm{~nm}$ and $500 \mathrm{GHz}$ Le Croy oscilloscope for the analysis of the pulse characteristics of the signal.

\section{Results and Discussion}

In order to investigate the continuous wave operation of the laser, the system is tested without the saturable absorber as to determine the minimum lasing threshold power, giving a value of $5.5 \mathrm{~mW}$. Next, the MWCNT-PVA saturable absorber is inserted into the laser cavity, giving a lasing threshold of about $8.1 \mathrm{~mW}$. Figure 4 shows the measured threshold of the laser with and without the saturable absorber as 8.1 and $5.5 \mathrm{~mW}$, respectively, taken from the lasing against pump power, with the corresponding slope efficiency of $6.2 \%$ and $7.4 \%$, respectively.

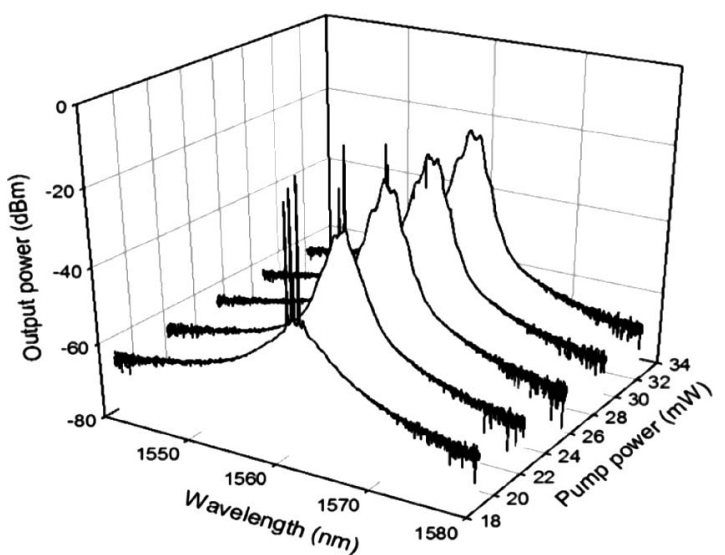

Fig. 5. Semi-logarithmic plot of the power versus wavelength of a single $Q$-switched output pulse for different pump powers. 


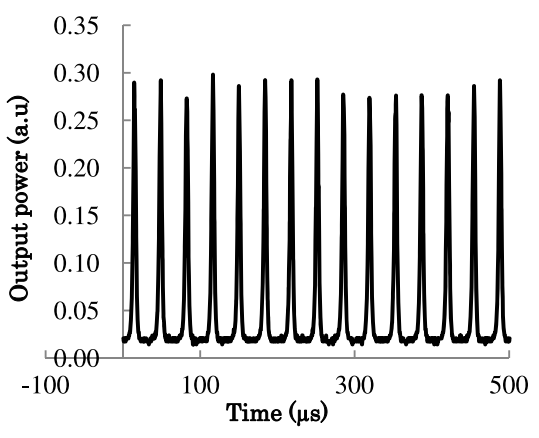

Fig. 6. Output power versus time of the repetitively $Q$-switched laser pumped of $32.2 \mathrm{~mW}$.

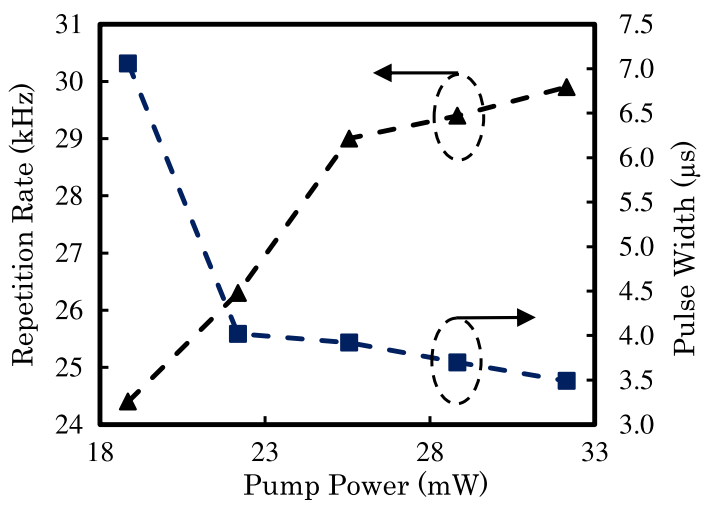

Fig. 7. Pulse repetition rate and pulse width against pump power.

The lower slope efficiency and the higher threshold for the laser system with the saturable absorber is expected due to absorption losses that occur when light travels into the saturable absorber. A stable self-starting $Q$-switched operation is achieved once the pump power reaches $18.8 \mathrm{~mW}$.

Figure 5 shows the evolution of $Q$-switched pulse laser spectra in accordance with the increase in pump power. As can be seen in Fig. 5, raising the pump power will affect the stability of $Q$ switched pulse generation. The output spectrum measurement is taken initially at the lasing threshold of the $Q$-switched operation, where multiple peaks of lasing can be observed. At this point, the recorded repetition rate of the pulse train is $24.4 \mathrm{kHz}$, which is measured by a photodetector with the bandwidth of $10 \mathrm{GHz}$. When the pump power is raised gradually to $32.15 \mathrm{~mW}$, the laser output exhibits spectral broadening with a central wavelength fixed at $1559.9 \mathrm{~nm}$ resulting in a stable pulse train with minimum fluctuation and steady repetition rate of $29.9 \mathrm{kHz}$. The temporal behavior of the pulse is recorded and shown in Fig. $\underline{6}$.

Figure 7 represents the dependency of repetition rate and pulse width on the pump power. Within this range of pump power, a stable $Q$-switched operation is observed. The repetition rate is detuned from 24.4 to $29.9 \mathrm{kHz}$ with the increase in pump power, confirming the intrinsic features of passive $Q$-switching of the laser caused by the saturable absorber. Higher pump power induces shorter time to refill the extracted energy between two successive pulses resulting in higher repetition rate. However, when the pump power is raised beyond $32.15 \mathrm{~mW}$, the $Q$ switching operation starts to become unstable with noticeable amplitude variations and the output power starts to decrease. The pump power is restricted not to be over this level to avoid optical power induced thermal damage on the saturable absorber.

The recorded pulse width at the threshold of repetitive $Q$-switched operation is approximately $7 \mu \mathrm{s}$. The pulse width is relatively stretched due to the long ring cavity, which subsequently gives rise to a long cavity lifetime. It can be further narrowed by replacing the EDF with a shorter length and a higher dopant concentration.

As presented in Fig. 8, with the increase of the pump power, the pulse width is narrowed to $3.49 \mu \mathrm{s}$ from $7.06 \mu \mathrm{s}$ and. Concurrently, it is also observed that the pulse amplitude increases, hence indicating the rise in the pulse energy. This result is consistent with the theory of saturable absorber passive $Q$-switching.

To investigate the correlation between the pump power and the pulse energy, we measured the average output power of the repetitive $Q$-switched pulses as a function of the pump power. The pulse energy is obtained by dividing mathematically the average
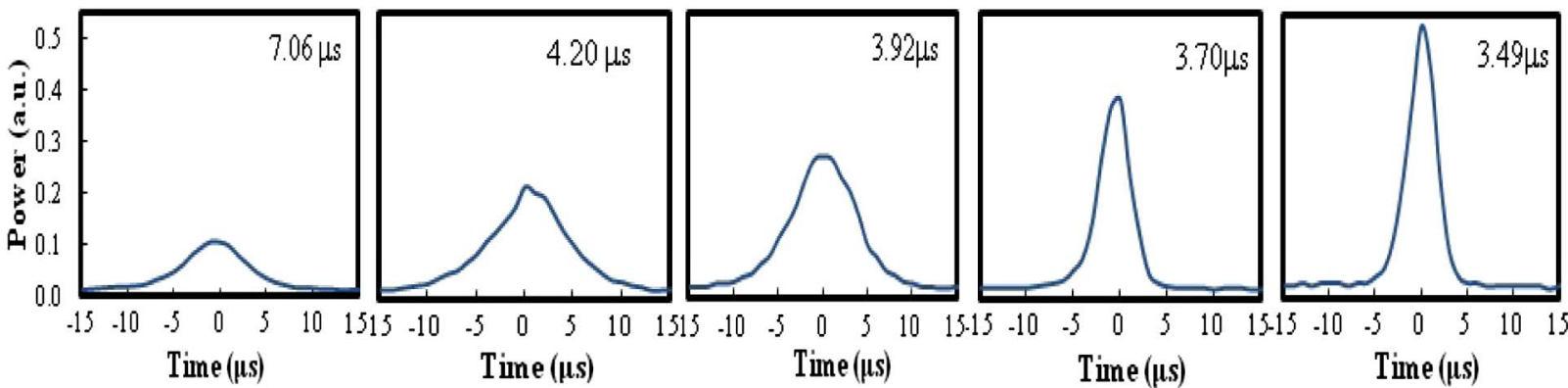

$$
\text { a) } \mathrm{Pp}=18.85 \mathrm{~mW}
$$

b) $\mathrm{Pp}=22.18 \mathrm{~mW}$

c) $\mathrm{Pp}=25.56 \mathrm{~mW}$

d) $\mathrm{Pp}=28.83 \mathrm{~mW}$

e) $\mathrm{Pp}=32.15 \mathrm{~mW}$

Fig. 8. Output power versus time for single repetitively $Q$-switched pulses obtained for different continuous pump powers $P / p$. The pulse widths (full width at half-maximum) obtained for each pulse are inset. (a) $P p=18.85 \mathrm{~mW}$, (b) $P p=22.18 \mathrm{~mW}$, (c) $P p=25.56 \mathrm{~mW}$, (d) $P p=28.83 \mathrm{~mW}$, (e) $P p=32.15 \mathrm{~mW}$ 


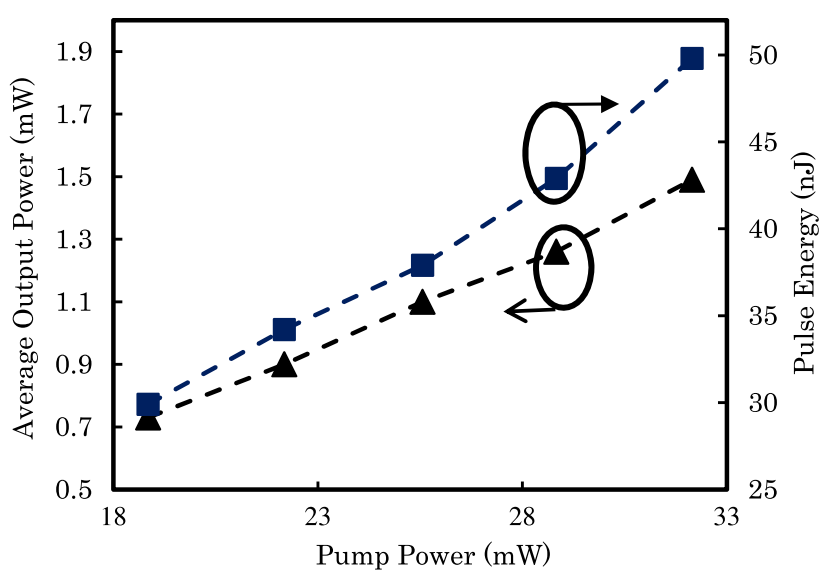

Fig. 9. Pulse energy $(\mathrm{nJ})$ and average output power $(\mathrm{mW})$ as functions of pump power.

output power by the corresponding repetition rate. As clearly shown in Fig. 9 , the average output power and pulse energy are found to rise linearly against the pump power, giving a maximum average output power of $1.49 \mathrm{~mW}$ and pulse energy of $49.8 \mathrm{~nJ}$ at maximum pump power of $32.15 \mathrm{~mW}$. The pulse energy gradually rises up linearly with the pump power within the detuning range.

\section{Conclusion}

In conclusion, we have demonstrated a passive and stable $Q$-switched pulse using a MWNTs-PVA thin film based saturable absorber. The film is sandwiched between two ferrules and the saturable absorber is incorporated in the EDFL ring cavity to generate a $Q$-switched laser operating in $1.5 \mu \mathrm{m}$ regions. The MWCNTs are introduced in the PVA host polymer, producing a smoother surface and more uniform thin film compared to PEO. Among many samples, the saturable absorber with the concentration of 1.25 wt. \%, of MWCNTs shows a consistency in generating pulsed laser with a good range of tunable repetition rate, shortest pulse width, and produces a high pulse energy and peak power. The improved average output power and pulse energy are also demonstrated using the saturable absorber. Besides showing good $Q$-switching performance, the MWCNTs PVA polymer composites-based saturable absorber is easy to fabricate and cheap. This makes the proposed saturable absorber a suitable component for generating a $Q$-switched laser in the $1.5 \mu \mathrm{m}$ wavelength region passively.

We would like to thank University of Malaya for providing the funding for this work under the grant RU002/2013.

\section{References}

1. S. Y. Set, H. Yaguchi, Y. Tanaka, and M. Jablonski, "Ultrafast fiber pulsed lasers incorporating carbon nanotubes," IEEE J. Sel. Top. Quantum Electron. 10, 137-146 (2004).

2. S. Yamashita, Y. Inoue, S. Maruyama, Y. Murakami, H. Yaguchi, M. Jabslonski, and S. Y. Set, "Saturable absorbers incorporating carbon nanotubes directly synthesized onto substrate and fibers and their application to mode locked fiber lasers," Opt. Lett. 29, 1581-1583 (2004).
3. S. Yamashita, Y. Inoue, K. Hsu, T. Kotake, H. Yaguchi, D. Tanaka, and S. Y. Set, "5-GHz pulsed fiber Fabry-Perot laser mode-locked using carbon nanotubes," IEEE Photon. Technol. Lett. 17, 750-752 (2005).

4. A. V. Tausenev, E. D. Obraztsova, A. S. Lobach, A. I. Chernov, V. I. Konov, P. G. Kryukov, and E. M. Dianov, "177 fs erbiumdoped fiber mode locked with a cellulose polymer film containing single-wall carbon nanotubes," Appl. Phys. Lett. 92, 171113 (2008).

5. A. V. Denisov, A. G. Kuznetsov, D. S. Kharenko, S. I. Kablukov, and S. A. Babin, "Frequency doubling and tripling in a Q-switched fiber laser," Laser Phys. 21, 277-282 (2011).

6. V. N. Fillippov, A. N. Starodumov, and A. V. Kiryanov, "All-fiber passively $Q$-switched low threshold erbium laser," Opt. Lett. 26, 343-345 (2001).

7. F. Wang, A. G. Rozhin, V. Scardaci, Z. Sun, F. Hennrich, I. H. White, W. I. Milne, and A. C. Ferrari, "Wideband-tuneable, nanotube mode-locked fiber laser," Nat. Nanotechnol. 3, 738-742 (2008).

8. H. B. Qin, Z. Zhuo, J. Liu, H. B. Zhang, and Y. G. Wang, "Diode pumped passively $Q$-switched $\mathrm{Nd}: \mathrm{YVO}_{4}$ laser with a carbon nanotube saturable absorber," Laser Phys. 21, 1562-1565 (2011).

9. T. R. Schibli, K. Minoshima, H. Kataura, E. Itoga, N. Minami, S. Kazaoui, K. Miyashita, M. Tokumoto, and Y. Sakakibara, "Ultrashort pulse generation by saturable absorber mirrors based on polymer-embedded carbon nanotubes," Opt. Express 13, 8025-8031 (2005).

10. J. C. Chiu, C. M. Chang, B. Z. Hsieh, S. C. Lin, C. Y. Yeh, G. R. Lin, and W. H. Cheng, "Pulse shortening mode-locked fiber laser by thickness and concentration product of carbon nanotube based saturable absorber," Opt. Express 19, 4036-4041 (2011).

11. K. N. Cheng, Y. H. Lin, S. Yamashita, and G. R. Lin, "Harmonic order-dependent pulse width shortening of a passively mode-locked fiber laser with a carbon nanotube saturable absorber," IEEE Photon. J. 4, 1542-1552 (2012).

12. K. N. Cheng, Y. H. Lin, and G. R. Lin, "Single-and doublewalled carbon nanotube based saturable absorbers for passive mode-locking of an erbium-doped fiber laser," Laser Phys. 23, 045105 (2013).

13. K. Ramudurai, C. L. Cromer, L. A. Lewis, K. E. Hurst, A. C. Dillon, R. L. Mahajan, and J. H. Lehman, "High-performance carbon nanotubes coatings for high power laser radiometry," J. Appl. Phys. 103, 013103 (2008).

14. F. Banhart, "Irradiation effects in carbon nanostructures," Rep. Prog. Phys. 62, 1181-1221 (1999).

15. F. Ahmad, H. Haris, R. M. Nor, N. R. Zulkepley, H. Ahmad, and S. W. Harun, "Passively $Q$-switched EDFL using a multiwalled carbon nanotube polymer composite based on a saturable absorber," Chin. Phys. Lett. 31, 034204 (2014).

16. L. Zhang, Y. G. Wang, H. J. Yu, S. B. Zhang, W. Sun, W. H. Xue, C. Lin, and J. M. Li, "Passively mode-locked Nd:YVO laser using a single-walled carbon nanotube saturable absorber pumped by $880 \mathrm{~nm}$ laser diode," Jpn. J. Appl. Phys. 50, 122703 (2011).

17. H. Yu, L. Zhang, Y. G. Wang, S. L. Yan, W. Sun, Y. Tsang, and $\mathrm{X}$. Lin, "Sub-100 ns solid-state laser $Q$-switched with double wall carbon nanotubes," Opt. Commun. 306, 128-130 (2013).

18. M. H. Christie and A. P. Nikolas, "Structure and applications of polyvinyl alcohol hydrogels produced by conventional cross linking or by freezing/thawing methods," Adv. Polym. Sci. 153, 37-65 (2000).

19. R. Saito, A. Jorio, A. G. Souza, G. Dresselhaus, M. S. Dresselhaus, and M. A. Pimenta, "Probing phonon dispersion relations of graphite by double resonance Raman scattering," Phys. Rev. Lett. 88, 027401 (2001).

20. J. Kurti, V. Zolyomi, A. Gruneis, and H. Kuzmany, "Double resonant Raman phenomena enhanced by van Hove singularities in single-wall carbon nanotubes," Phys. Rev. B 65, 165433 (2002).

21. N. Chakrapani, S. Curran, B. Q. Wei, P. M. Ajayan, A. Carrillo, and R. S. Kane, "Spectral fingerprinting of structural defects in plasma-treated carbon nanotubes," J. Mater. Res. 18, 25152521 (2003). 